\title{
Ginsenoside Rb1 inhibits neuronal apoptosis and damage, enhances spinal aquaporin 4 expression and improves neurological deficits in rats with spinal cord ischemia-reperfusion injury
}

\author{
FEI HUANG ${ }^{1}$, YA-NAN LI ${ }^{2}$, FEI YIN ${ }^{1}$, YUN-TAO WU ${ }^{1}$, DONG-XU ZHAO ${ }^{1}$, \\ YE $\mathrm{LI}^{1}$, YUN-FENG ZHANG ${ }^{1}$ and QING-SAN ZHU ${ }^{1}$ \\ ${ }^{1}$ Department of Orthopedics, China-Japan Union Hospital of Jilin University, Changchun, Jilin 130033; \\ ${ }^{2}$ Department of Pediatrics, The First Hospital of Jilin University, Changchun, Jilin 130021, P.R. China
}

Received March 5, 2014; Accepted December 2, 2014

DOI: $10.3892 / \mathrm{mmr} .2015 .3162$

\begin{abstract}
Ginsenoside Rb1 is a potential therapeutic agent for the treatment of spinal cord ischemia-reperfusion injury (SCII), although it has not yet been investigated in depth. The aim of the present study was to investigate the effects of ginsenoside Rb1 treatment on SCII and aquaporin-4 (AQP4) expression in the rat spinal cord. Experimental animals were subjected to one of four conditions, including the blank control condition, sham procedure, spinal cord ischemia-reperfusion induced by abdominal aortic occlusion or spinal cord ischemia-reperfusion followed by ginsenoside $\mathrm{Rb} 1$ treatment. Locomotor activity was evaluated using the Basso Beattie Bresnahan scale. Spinal cord damage was assessed with hematoxylin and eosin and Nissl staining and the apoptotic rate was measured using terminal deoxynucleotidyl transferase dUTP nick end labeling. AQP4 expression was assessed by immunohistochemical analysis, western blotting and reverse transcription-quantitative polymerase chain reaction. Abdominal aortic occlusion resulted in the reduced expression of AQP4 in the spinal cord, which gradually recovered over time. Furthermore, ginsenoside Rb1 treatment significantly attenuated this decrease and protected the integrity of and reduced the apoptotic rate in spinal cord neurons. Treatment with ginsenoside Rb1 attenuated the initial downregulation and advanced the recovery of AQP4 expression levels, suggesting a possible mechanism for the therapeutic effects on SCII.
\end{abstract}

\section{Introduction}

Spinal cord ischemia-reperfusion injury (SCII) is a secondary injury resulting from the temporary interruption of blood

Correspondence to: Dr Qing-San Zhu, Department of Orthopedics, China-Japan Union Hospital of Jilin University, 126 Xiantai Street, Changchun, Jilin 130033, P.R. China

E-mail: hflyn2011@sina.com

Key words: spinal cord damage, ginsenoside rb1, aquaporin-4 supply to the spinal cord and may result in irreversible vascular damage with subsequent paraplegia or other neurological deficits (1). This severe complication is frequently observed in transient ischemic insults of the spinal cord and following surgical repair of thoracoabdominal aortic aneurysms (2). While the exact mechanism of secondary SCII remains to be elucidated, there are multiple contributing cellular responses, including overproduction of reactive oxygen species and oxygen free radicals that lead to excessive lipid peroxidation, protein and DNA damage, neuronal apoptosis and calcium overload (3-5). Several drugs are currently used for the treatment of SCII, including glucocorticoids, neurotrophic factors and nerve regeneration factors. However, a detailed understanding of the effects of these drugs is lacking (6). Further investigation into the mechanism of SCII may assist in providing novel therapeutic targets.

Edema of the spinal cord following the initial injury may be an important contributing factor to the development of SCII, posing a major challenge clinically, as it is often long-lasting and therapy resistant. The transport of water across cellular membranes contributes to the development of such edematous conditions, thus proteins involved in this process may present therapeutic targets. Aquaporin-4 (AQP4) is the most abundant member of a family of water-conducting integral membrane proteins that is highly expressed in glial cells of the spinal cord $(7,8)$. AQP4 expression parallels the changes in water content in the injured spinal cord (9) and has been observed to be directly involved in spinal cord injury-associated edema (10). Furthermore, AQP4 has been implicated in spinal cord damage in multiple sclerosis (11) and amyotrophic lateral sclerosis (12). Thus, the development of an effective and safe therapeutic agent targeting AQP4 expression may be beneficial for the treatment of SCII.

A commonly used traditional remedy for numerous medical conditions, including central nervous system and neurodegenerative disorders, is ginseng (13). While the active agent remains to be elucidated, it is hypothesized to be ginsenoside, an extract from the stem and leaf of ginseng. Currently, $>50$ different ginsenoside monomers have been isolated (14-16), each with different effects. Ginsenoside Rg has an anti-fatigue effect and is able to improve memory and learning ability $(15,17)$. 
Ginsenoside $\operatorname{Rg} 2$ is also able to improve myocardial ischemia and hypoxia in the treatment and prevention of coronary heart disease $(18,19)$. Ginsenoside $\mathrm{Rg} 3$ can promote tumor cell apoptosis and inhibit tumor cell growth $(20,21)$. Ginsenoside Rb2 acts as an antioxidant, removing free radicals and improving recovery from myocardial ischemic-reperfusion injury (22). Ginsenoside Rh1 suppresses the central nervous system and may have an hypnotic effect (23). However, the most abundant and representative single monomer, ginsenoside Rb1, has demonstrated therapeutic potential for a variety of conditions. Several studies have revealed that the Rb1 monomer is anti-apoptotic $(24,25)$ and has the ability to alleviate oxidative stress by scavenging oxygen free radicals (26), blocking calcium channels (27) and attenuating ischemic-reperfusion injury $(28,29)$. As there is evidence that ginsenoside Rb1 may reduce the damaging effects of myocardial and cerebral ischemia, it was hypothesized that it may also have a protective effect against SCII. Therefore, the effect of ginsenoside Rb1 treatment on abdominal aortic occlusion-induced SCII was examined. Furthermore, the expression of AQP4 was assessed as a potential mechanism for the neuroprotective effects of ginsenoside $\mathrm{Rb} 1$ treatment.

\section{Materials and methods}

Animals. A total of 96 male and female 8-10 week-old Sprague-Dawley rats (weighing 220-260 g) were obtained from the animal experimental center of Jilin University (Changchun, China) and maintained in an automatic light/ dark cycle with ad libitum access to food and water. Rats were acclimated for 1 week prior to experimentation. Animal care and procedures were approved by the Ethics Committee of Jilin University (Changchun, China).

Drug treatment and dosage. Ginsenoside $\mathrm{Rb} 1\left(\mathrm{C}_{54} \mathrm{H}_{92} \mathrm{O}_{23}\right.$, molecular weight: $1109.29 \mathrm{Da}$ ) was provided by the Department of Organic Chemistry, Jilin University. The appropriate dosage of ginsenoside $\mathrm{Rb} 1$ for rats was established with preliminary experiments to determine the minimum effective concentration of ginsenoside Rb1 following SCII in rats. Concentrations of ginsenoside $\mathrm{Rb} 1>10 \mathrm{mg} / \mathrm{kg} /$ day exhibited toxicity in rat organs. Therefore, the maximum dosage of $10 \mathrm{mg} / \mathrm{kg} /$ day was administered as a once-daily intraperitoneal (IP) injection in the present experiments.

Spinal cord ischemic-reperfusion injury model. Animals were randomly assigned to one of the four following groups with a total of 24 animals in each group: i) Blank control condition, ii) sham surgery procedure; iii) SCII induced by abdominal aortic occlusion control group or iv) SCII group treated with ginsenoside Rb1. All rats were anesthetized with an IP injection of $10 \%$ chloral hydrate. The model of transient SCII by an abdominal aortic occlusion was implemented according to a previously reported method (30). Briefly, animals were anesthetized and body temperature was maintained at $37^{\circ} \mathrm{C}$ with a heating pad. The rats were placed in a right lateral position and the abdomen was opened through a midline incision to expose the abdominal aorta. The aorta and its branches were meticulously dissected from the caudal to the left renal artery. A microvessel clamp was placed across the aorta just distal to the left renal artery to occlude blood supply to the lumbar spinal cord. Following aortic declamping, the abdominal wall was closed and the animal was permitted to regain consciousness. Transient spinal cord ischemia was induced for $60 \mathrm{~min}$, followed by $60 \mathrm{~min}$ of reperfusion. In the sham group, the animals underwent the laparotomy only, with no aortic occlusion. In the SCII control group, an IP injection of $0.9 \%$ sodium chloride was administered, whereas in the treatment group, a dose of $10 \mathrm{mg} /$ $\mathrm{kg} /$ day of ginsenoside Rb1 was administered after $60 \mathrm{~min}$ of reperfusion. For each group, rats were sacrificed at 1, 3, 5 and 7 days after the procedure ( $n=6$ per time-point) and the spinal cord was quickly removed for further analyses.

Behavioral analysis of spinal cord injury. To assess neurological deficits, locomotor activity was examined and measured in all rats using the Basso Beattie Bresnahan (BBB) rating scale (31). This commonly used method for assessment of recovery from spinal cord injury provides a measure of hindlimb nerve function by rating activity on a scale between 0 and 21 , with a score of 21 indicating full recovery.

Morphological analysis. Spinal cord specimens were embedded in paraffin and sectioned at $4 \mu \mathrm{m}$. Sections from the lumbar (L3) level of each spinal cord were deparaffinized and underwent hematoxylin and eosin $(\mathrm{H} \& \mathrm{E})$ or Nissl staining. They were subsequently mounted on slides and coverslipped with Permount (Thermo Fisher Scientific, Waltham, MA, USA). Neuronal injury, cell morphology and Nissl bodies were observed and evaluated under a light microscope (magnification, x400; CX21; Olympus Corp., Tokyo, Japan).

Quantification of apoptosis using the terminal deoxynucleotidyl transferase dUTP nick end labeling (TUNEL) technique. Paraffin-embedded spinal cord specimens were sectioned $(4 \mu \mathrm{m})$ and deparaffinized. Sections were then treated with a TUNEL reaction mixture (Roche, Basel, Switzerland) for $60 \mathrm{~min}$ at $37^{\circ} \mathrm{C}$ followed by a $60 \mathrm{~min}$ incubation at $37^{\circ} \mathrm{C}$ in a Converter-POD solution (Roche) and reacted with 3,3'-diaminobenzidine for visualization. Apoptotic cells were observed with a light microscope (magnification, x400) and appeared as cells with brown stained nuclei, with normal cells appearing blue. Six sections from each group were examined, with $\sim 500$ cells counted on each section. The apoptotic rate was calculated based on the number of TUNEL-positive cells out of the total number counted.

Analysis of AQP4 protein level by western blotting. Protein levels from spinal cord extracts were analyzed using western blotting. Total protein was extracted from the spinal cord tissue and $50 \mu \mathrm{g}$ of the extract from each isolate was separated on a $10 \%$ SDS-PAGE gel and transferred onto a nitrocellulose filter (Sartorius, Goettingen, Germany) for immunodetection. The filters were incubated with rabbit anti-rat polyclonal AQP4 $(1: 1,000$, ab46182) or $\beta$-actin $(1: 1,000$; ab8227) antibodies (Abcam, Cambridge, UK), followed by horseradish peroxidase-conjugated goat anti-rabbit polyclonal IgG (1:800; sc2004; Santa Cruz Biotechnology, Inc., Dallas, TX, USA). Immunoreactive bands were visualized with enhanced chemiluminescence (Santa Cruz Biotechnology, Inc.) followed by optical density analysis. 
Analysis of AQP4 protein level using immunohistochemistry. Antigen retrieval was performed by incubating spinal cord sections in a citrate-buffered saline solution $\left(\mathrm{pH} \mathrm{6.0)}\right.$ at $98^{\circ} \mathrm{C}$ for $10 \mathrm{~min}$. Subsequently, the sections were allowed to cool, then rinsed for $3 \mathrm{~min}$ in phosphate-buffered saline (PBS) three times, incubated with $1 \%$ Triton X-100 in PBS for 20 min and then incubated with goat serum at $37^{\circ} \mathrm{C}$ for $60 \mathrm{~min}$. Sections were rinsed three times with PBS, followed by a $12 \mathrm{~h}$ incubation with rabbit anti-rat polyclonal AQP4 antibody (1:500; ab46182) and a 60 min incubation in goat anti-rabbit IgG heavy and light chain DyLight ${ }^{\circledR} 488$ polyclonal secondary antibody (1:2,000; ab96899; Abcam). Staining was visualized by fluorescence microscopy (BX51TF; Olympus Corp.) and quantified by measuring the integrated optical density using Image-Pro Plus 6.0 imaging software (Media Cybernetics Inc., Rockville, MD, USA).

Analysis of AQP4 mRNA levels. Expression of AQP4 mRNA was measured in spinal cord tissue samples using reverse transcription-quantitative polymerase chain reaction (RT-qPCR). Total RNA was extracted from spinal cord tissue with TRIzol reagent (Invitrogen Life Technologies, Carlsbad, CA, USA) according to the manufacturer's instructions. RNA was converted into cDNA with M-MLV reverse transcriptase (Promega Corporation, Madison, WI, USA). Quantitative detection of transcripts was performed by RT-PCR with SYBR Green using the following gene-specific primers for AQP4 and rat $\beta$-actin: AQP4, sense 5'-ATT GGG AGT CAC CAC GGT TCA T-3' and antisense 5'-TGG ATT CAT GCT GGC TCC GGT AT-3' (211 bp product) and $\beta$-actin, sense 5'-TCA CCC ACA CTG TGC CCA TCT ACG-3' and antisense 5'-GGA TGC CAC AGG ATT CCA TAC CCA-3' (343 bp product). A total of 40 amplification cycles were performed, consisting of $94^{\circ} \mathrm{C}$ for $30 \mathrm{sec}, 58^{\circ} \mathrm{C}$ (for $\mathrm{AQP} 4$ ) or $57^{\circ} \mathrm{C}$ (for $\beta$-actin) for $30 \mathrm{sec}$ and $72^{\circ} \mathrm{C}$ for $40 \mathrm{sec}$. The results were analyzed using the $2^{-\Delta \Delta C \mathrm{~T}}$ method (32) and values were normalized and expressed relative to the blank group levels.

Statistical analysis. Data are presented as the mean \pm standard deviation or standard error of the mean. Differences between groups were assessed by analysis of variance using SPSS 14.0 statistics software (SPSS, Inc., Chicago, IL, USA). P $<0.05$ was considered to indicate a statistically significant difference.

\section{Results}

Ginsenoside Rb1 enhances hindlimb nerve function in SCII rats. The BBB scores for the blank group and the sham group were 21. The BBB scores of the untreated SCII group and ginsenoside Rb1-treated SCII group (treatment group) at day 1 were $2.34 \pm 0.20$ and $2.62 \pm 0.36$, respectively, and then gradually recovered reaching $15.42 \pm 1.36$ and $18.73 \pm 1.55$, respectively, at day 7. BBB scores from the untreated SCII group were lower than those of the sham group at all time points $(\mathrm{P}<0.05)$ and the BBB scores of the treatment group were higher than those of the untreated SCII group at all time points $(\mathrm{P}<0.05$; Fig. 1).

Ginsenoside Rb1 treatment alleviates neuronal damage in SCII rats as observed by $H \& E$ staining. Microscopic analysis of $\mathrm{H} \& \mathrm{E}$ stained spinal cord sections revealed normal

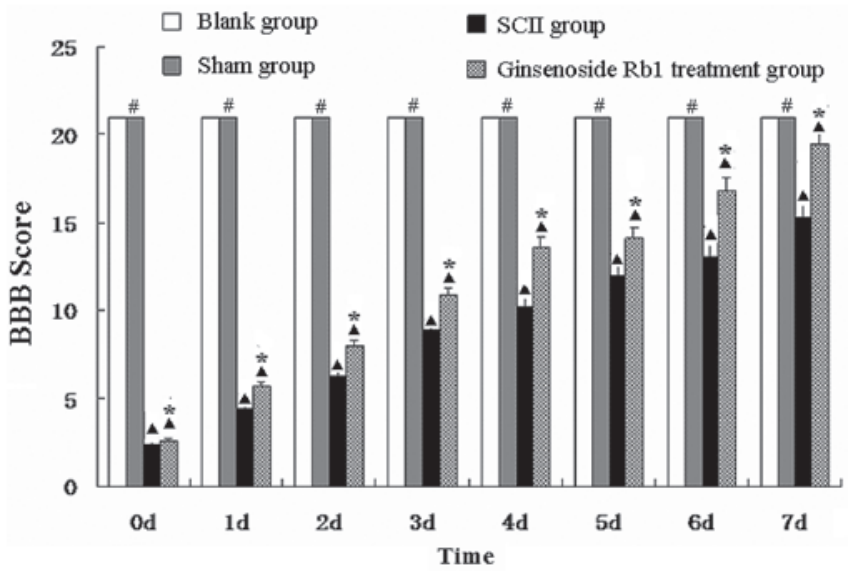

Figure 1. BBB locomotor rating scale values of rats in all experimental groups. ${ }^{\wedge} \mathrm{P}<0.05$ vs. blank group; ${ }^{*} \mathrm{P}<0.05$ vs. $\mathrm{SCII}$ group; ${ }^{*} \mathrm{P}>0.05$ vs. blank group. BBB, Basso Beattie Bresnahan; SCII, spinal cord ischemia-reperfusion injury.

morphology and clear cell structure in the blank and sham group specimens. Certain neurons in the untreated SCII group were damaged and enlarged, with pyknotic cell nuclei and tissue interstitial hyperemia. These features were not observed in specimens from ginsenoside Rb1-treated rats (Fig. 2).

Ginsenoside Rbl treatment alleviates neuron damage in SCII rats as observed by Nissl staining. Microscopic analysis of Nissl stained spinal cord sections demonstrated that the neurons in the blank and sham control groups were morphologically normal. In sections from the untreated SCII group, there was a decrease in the number of neurons and Nissl body structure was unclear, loose and disorganized. In addition, there was evidence of cytoplasmic condensation and marginalization, as well as signs of neuron enlargement and unclear nuclear structure. These appearances are indicative of cell and tissue damage and were not observed in sections from ginsenoside Rb1-treated rats (Fig. 3).

Ginsenoside Rb1 reduces apoptosis in SCII rats. Following TUNEL staining of spinal cord sections, the number of apoptotic cells was counted and the rate of apoptosis was calculated based on the total number of cells (Fig. 4A). The apoptotic rates of the blank $(17.68 \pm 1.15 \%)$ and sham $(18.21 \pm 1.26 \%)$ groups were lower than those from the untreated $(67.91 \pm 3.67 \%)$ and ginsenoside Rb1-treated $(54.36 \pm 3.26 \%)$ SCII groups at day $1(\mathrm{P}<0.05)$. The apoptotic rate of the treatment group was significantly lower than that of the untreated SCII group $(\mathrm{P}<0.05)$. Ginsenoside $\mathrm{Rb} 1$ treatment decreased the apoptotic rate of SCII between $44.38 \pm 3.17$ and $26.96 \pm 1.44 \%$ at day 7 $(\mathrm{P}<0.05)$, however, remained higher than that of the blank control group $(18.82 \pm 1.16 \%$; $\mathrm{P}<0.05$; Fig. 4B).

Ginsenoside Rb1 attenuates the decrease in AQP4 protein expression in SCII rats as measured by western blotting. Evaluation of AQP4 protein expression by western blotting revealed that protein levels in the blank and sham control groups were consistent and no significant differences were detected, suggesting that the sham injury had no effect on spinal cord AQP4 protein levels. Expression of AQP4 protein 


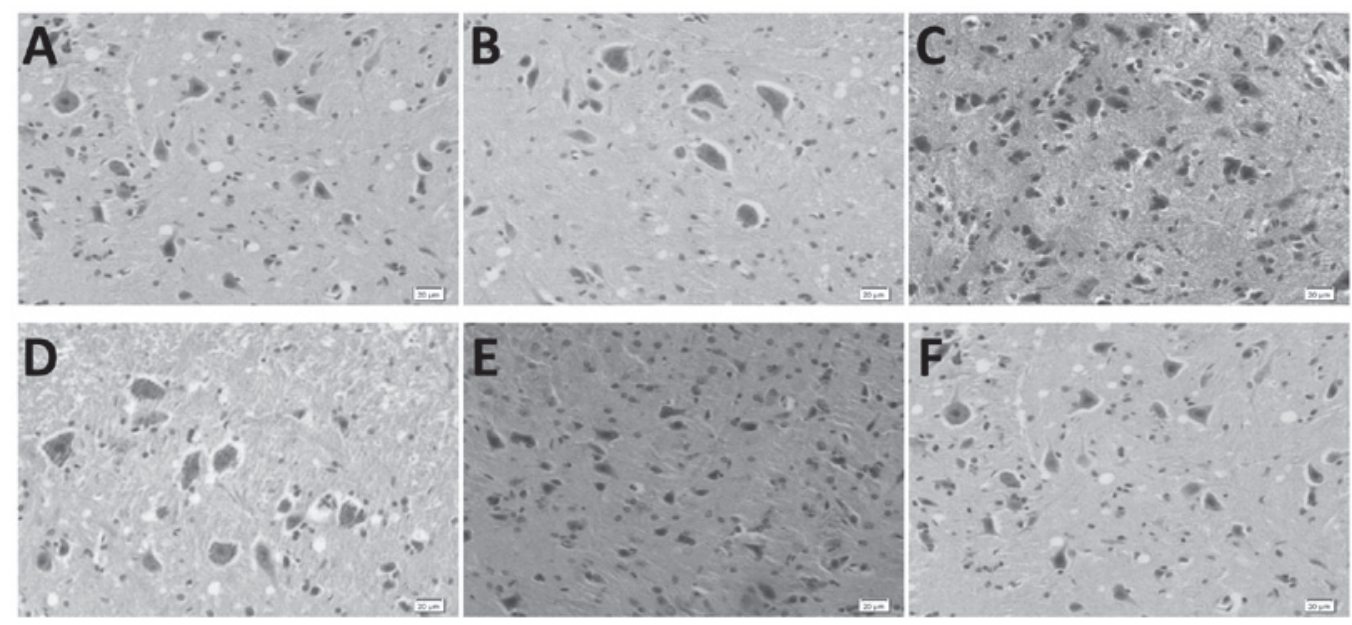

Figure 2. Hematoxylin and eosin staining of rat spinal cord tissue. (A) Blank group, (B) sham group, (C) SCII group (day 1), (D) treatment group (day 1), (E) SCII group (day 7) and (F) treatment group (day 7; magnification, x400). SCII, spinal cord ischemia-reperfusion injury.
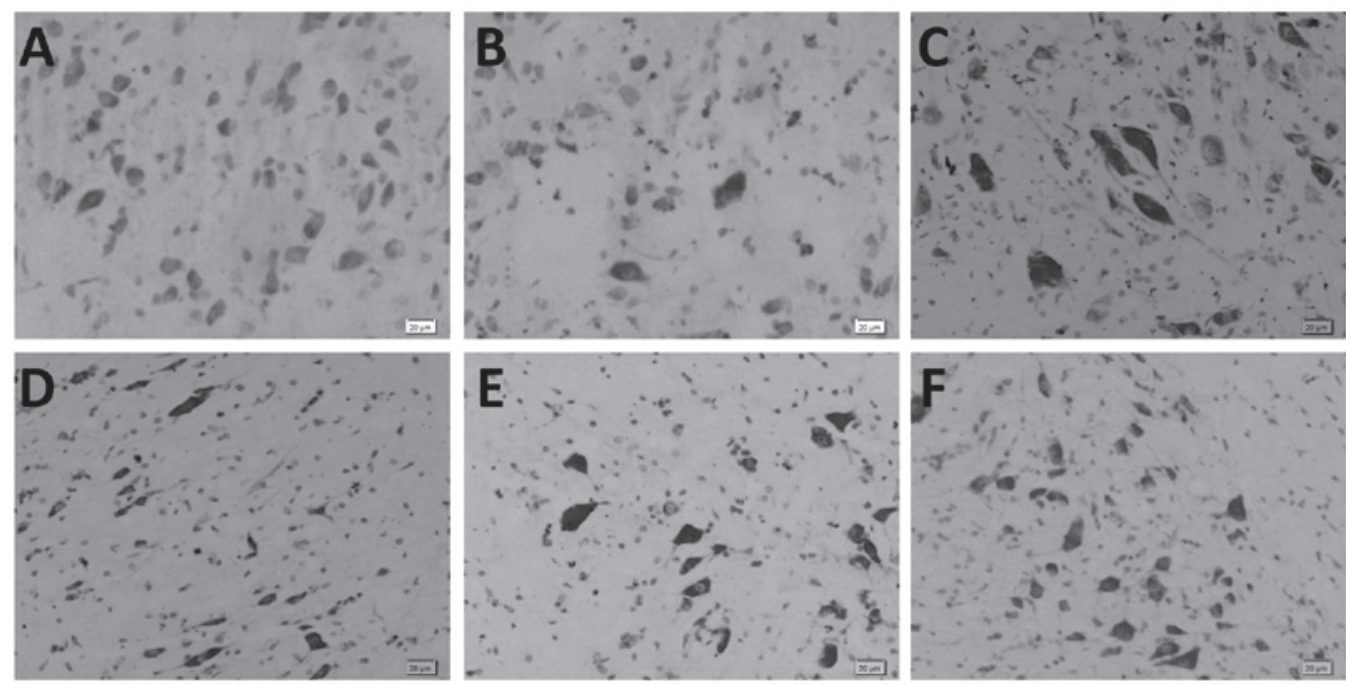

Figure 3. Nissl staining of rat spinal cord tissue. (A) Blank group, (B) sham group, (C) SCII group (day 1), (D) treatment group (day 1), (E) SCII group (day 7), (F) treatment group (day 7; magnification, $\mathrm{x} 400$ ). SCII, spinal cord ischemia-reperfusion injury.

in the untreated SCII group was significantly reduced to one third of the level of the blank group and gradually increased at days 3, 5 and 7 after the procedure. Although the level at day 7 increased to $79.9 \%$ of the level of the blank group, it remained lower than the levels identified in the blank and sham groups at days 3, 5 and $7(\mathrm{P}<0.05)$. Treatment with ginsenoside $\mathrm{Rb} 1$ for 1 day significantly increased AQP4 protein expression compared with the untreated SCII group $(\mathrm{P}<0.05)$. While protein expression in the treated SCII group was lower at day 1 compared with the blank and sham control groups, it gradually increased after 3 and 5 days and recovered to the blank control group level by day 7 (Fig. 5).

Ginsenoside Rb1 attenuates the decrease in AQP4 protein expression in SCII rats as measured by immunohistochemistry. Immunohistochemical analysis of AQP4 expression in spinal cord sections identified no significant difference between the blank and sham control groups. AQP4 immunofluorescence in the untreated SCII group was significantly decreased to $36.9 \%$ of the blank group level at day $1(\mathrm{P}<0.05)$. AQP4 immunofluorescence gradually increased to $84.6 \%$ of the blank group level at day 7 , however, remained lower than the blank and sham control groups $(\mathrm{P}<0.05)$. Treatment with ginsenoside Rb1 increased the quantity of AQP4 immunofluorescence compared with the untreated SCII group at all time points measured $(\mathrm{P}<0.05)$, but remained significantly lower than the blank group on day 1,3 and $5(\mathrm{P}<0.05)$. However, after 7 days, levels of AQP4 immunofluorescence were similar to those of the blank control group (Fig. 6).

Ginsenoside Rbl attenuates the decrease in AQP4 transcription in SCII rats. Alterations in the expression levels of AQP4 transcripts following SCII were similar to the changes observed at the protein level. The mRNA expression of AQP4 was not significantly different between the sham and blank control groups. AQP4 mRNA levels were lower in the untreated SCII group compared with the blank group and while levels increased over time, they remained lower than the blank group 


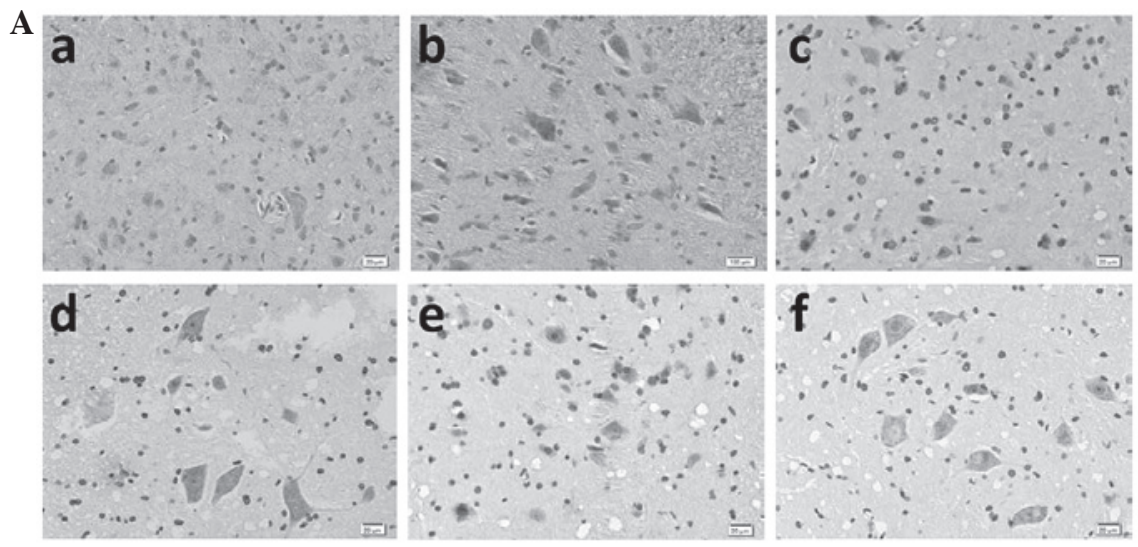

B

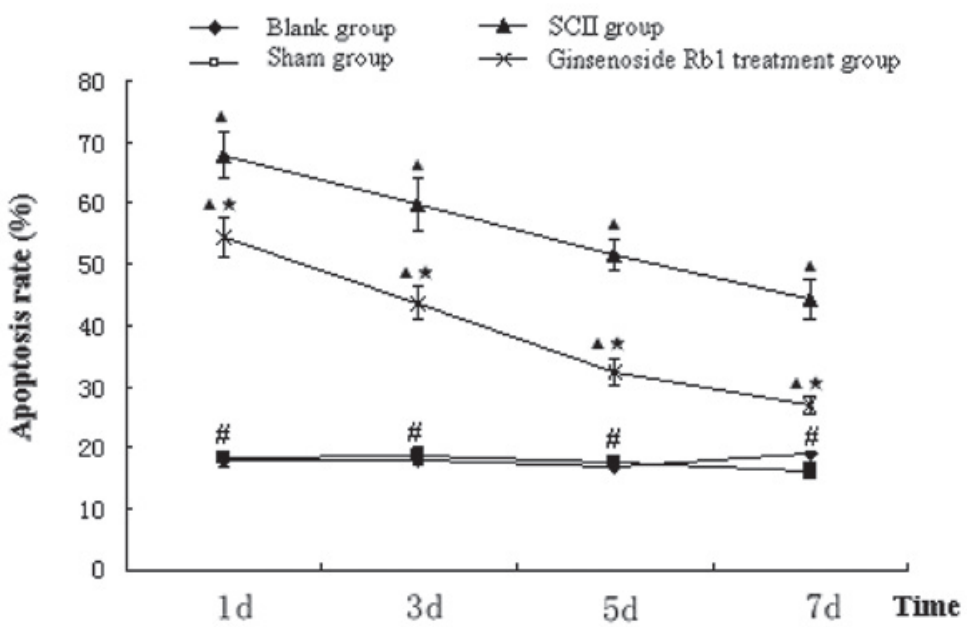

Figure 4. Apoptosis in SCII rat spinal cord. (A) Terminal deoxynucleotidyl transferase dUTP nick end labeling staining in rat spinal cord tissue: (Aa) Blank group (Ab) sham group, (Ac) SCII group (day 1), (Ad) treatment group (day 1), (Ae) SCII group (day 7) and (Af) treatment group (day 7; magnification, x400). (B) Quantification of apoptotic rate in SCII rat spinal cord. ${ }^{\mathbf{A}} \mathrm{P}<0.05$ vs. blank group; "P $<0.05$ vs. SCII group; ${ }^{\text {P }}>0.05$ vs. blank group. SCII, spinal cord ischemia-reperfusion injury.

A

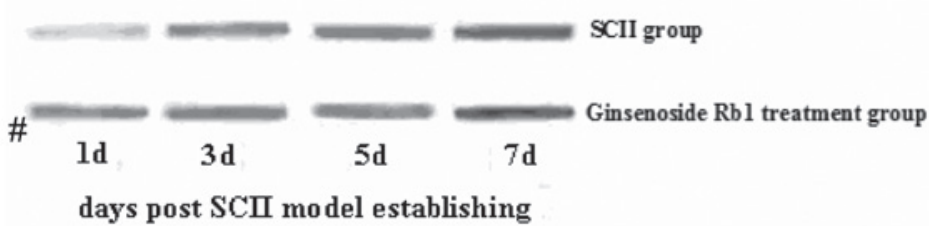

B

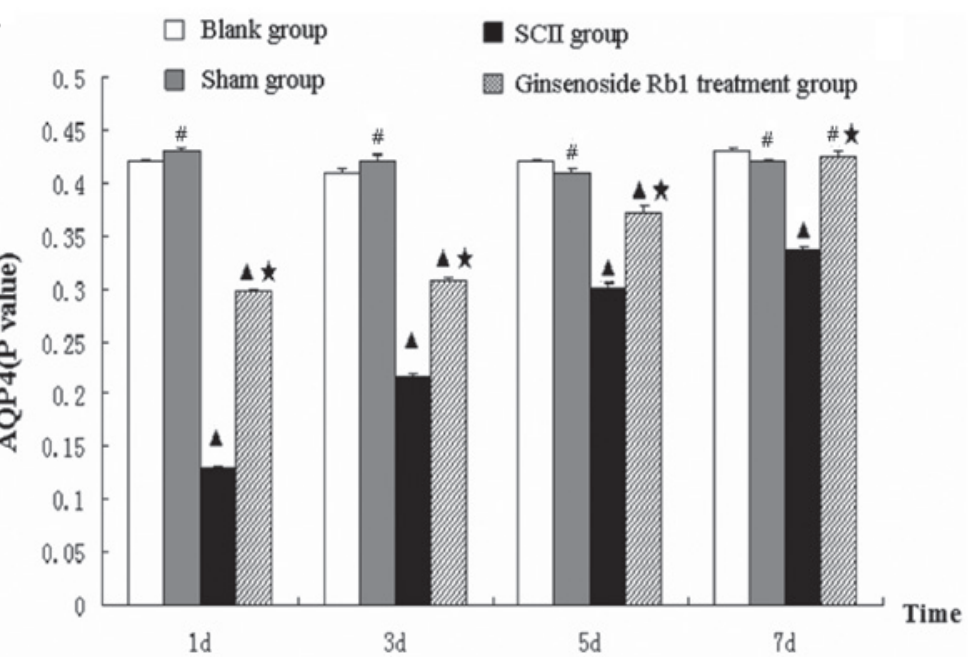

Figure 5. Western blot analysis of AQP4 protein expression in rat spinal cord tissue. (A) Representative image of western blotting. (B) Quantification of western blotting. ${ }^{\wedge} \mathrm{P}<0.05$ vs. blank group; ${ }^{\mathrm{P}}<0.05$ vs. SCII group; ${ }^{*} \mathrm{P}>0.05$ vs. blank group. AQP4, aquaporin-4; SCII, spinal cord ischemia-reperfusion injury. 
A
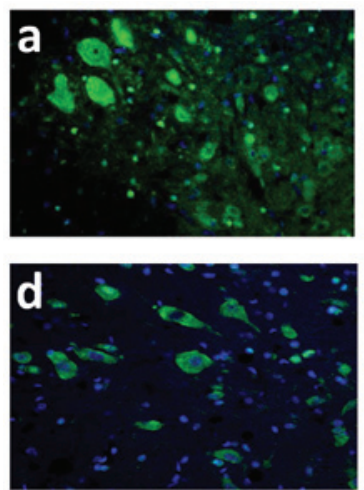
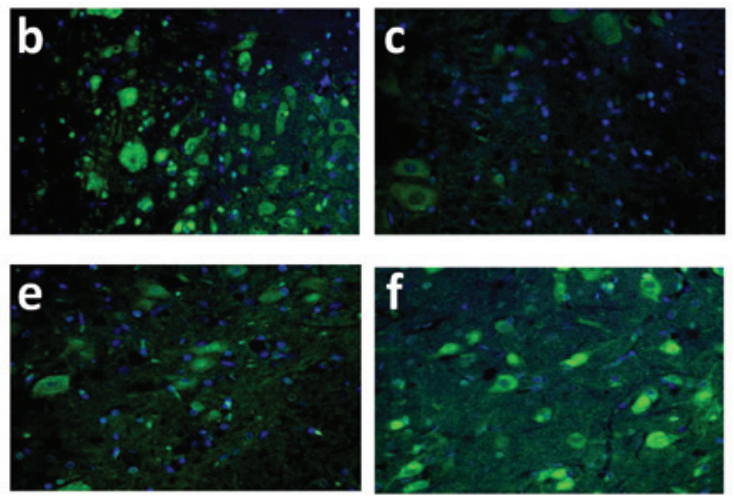

B

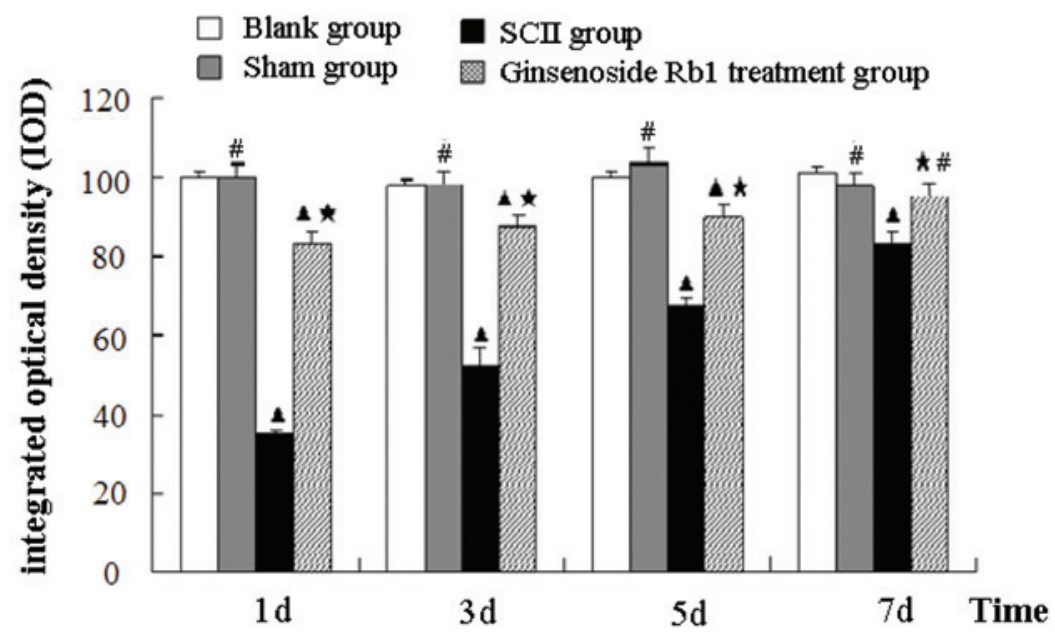

Figure 6. Immunohistochemical analysis of AQP4 protein expression in rat spinal cord tissue. (A) Microscopic images of AQP4 immunohistochemistry: (Aa) Blank group, (Ab) sham group, (Ac) SCII group (day 1), (Ad) treatment group (day 1), (Ae) SCII group (day 7), (Af) treatment group (day 7; magnification, $\mathrm{x} 400$ ). (B) Quantification of AQP4 immunofluorescence. ${ }^{\mathbf{A}} \mathrm{P}<0.05$ vs. blank group; ${ }^{\mathrm{P}}<0.05$ vs. SCII group; ${ }^{*} \mathrm{P}>0.05$ vs. blank group. AQP4, aquaporin-4; SCII, spinal cord ischemia-reperfusion injury.

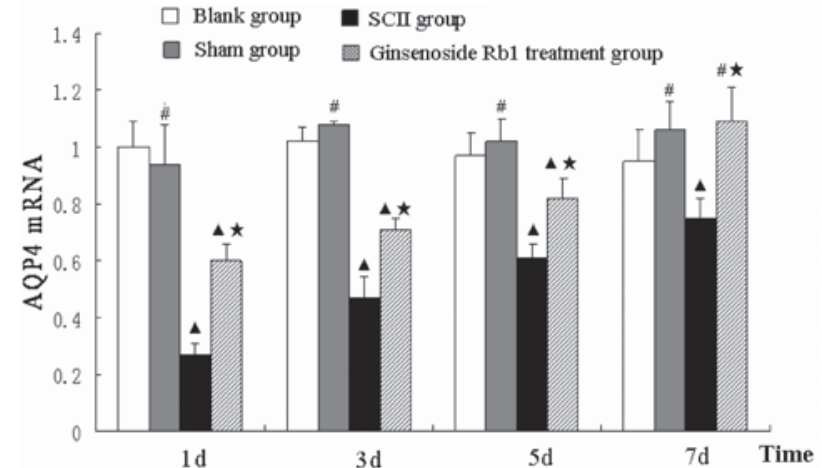

Figure 7. Quantification of AQP4 mRNA levels in rat spinal cord tissue. ${ }^{\wedge} \mathrm{P}<0.05$ vs. blank group; ${ }^{\mathrm{P}} \mathrm{P}<0.05$ vs. $\mathrm{SCII}$ group; ${ }^{*} \mathrm{P}>0.05$ vs. blank group. $\mathrm{AQP} 4$, aquaporin-4; SCII, spinal cord ischemia-reperfusion injury.

at day $7(\mathrm{P}<0.05)$. Treatment with ginsenoside $\mathrm{Rb} 1$ attenuated the decrease in AQP4 mRNA levels in SCII rats at day 1, 3 and 5 and promoted the recovery to control group levels by day 7 (Fig. 7).

\section{Discussion}

AQP4 is a transmembrane protein widely distributed throughout the spinal cord that acts as a bidirectional transporter facilitating the movement of water molecules through the cell membrane. A disruption in AQP4 expression may lead to a dysregulation of water transport in regions of high expression, including the spinal cord, resulting in edema. Spinal cord edema is one of the most important factors contributing to SCII and the degree of edema is associated with SCII prognosis $(9,33)$. Previous studies have found that the expression of AQP4 is decreased in spinal cord injury, which may result in acute spinal cord edema, ultimately affecting the normal function of the spinal cord $(9,34)$. While the exact mechanism of SCII remains to be elucidated, spinal cord edema occurs at an early phase and therefore the effects of AQP4 expression in the development of edema and SCII are uncertain. However, it is known that without early intervention, water imbalance and cell edema may lead to cell necrosis and apoptosis (35). Therefore the maintenance of proper cellular water balance is crucial and the identification of mechanisms contributing to this is of importance. Therefore, alterations in the expression of AQP4 may impart a mechanism for the development of SCII and thus provide a novel target for therapeutic intervention.

The present study found that AQP4 mRNA and protein expression was reduced following SCII in rats. The levels of AQP4 expression increased over time, but remained significantly lower than blank and sham control groups up to 7 days after the insult. Concomitant with the initial reduction in 
AQP4 expression was a loss of nerve function as evidenced by locomotor scores. In addition, as recovery of nerve function was accompanied by an increase in AQP4 protein expression, an association between AQP4 expression and normal nerve function was indicated. Therefore, it was suggested that manipulations or treatments resulting in an increase in AQP4 expression may provide a viable therapeutic intervention to increase functional recovery and prognosis following SCII.

As treatment with ginsenoside $\mathrm{Rb} 1$ has previously been demonstrated to have therapeutic potential in the treatment of ischemic injuries, its effect on SCII was investigated. Treatment with ginsenoside Rb1 significantly increased locomotor scores in rats subjected to SCII. In addition, morphological analysis of spinal cords revealed that ginsenoside Rb1 treatment significantly reduced signs of cellular damage caused by SCII, as well as significantly reducing levels of neuronal apoptosis. Finally, the current results demonstrated that SCII markedly reduced AQP4 expression and treatment with ginsenoside Rb1 attenuated this reduction, promoting recovery to near-normal levels by 7 days post-SCII. Therefore, the reduction in AQP4 expression caused by SCII may be partially corrected with ginsenoside $\mathrm{Rb} 1$ treatment.

In conclusion, the results demonstrate that SCII involves the abnormal expression of AQP4 and that treatment with ginsenoside Rb1 inhibits this change, improves impaired nerve function and prevents abnormalities in spinal cord nerve cell morphology. The present results provide a mechanism and new therapeutic option for the clinical treatment of SCII. Further investigation into the safety and efficacy of ginsenoside Rb1 treatment is required, including investigation into other clinical therapeutic approaches based on Traditional Chinese Medicine.

\section{Acknowledgements}

This study was sponsored by a grant awarded to Dr Fei Huang from the National Natural Science Foundation of China (grant no. 81301034$)$.

\section{References}

1. Wang JY, Shen J, Gao Q, et al: Ischemic postconditioning protects against global cerebral ischemia/reperfusion-induced injury in rats. Stroke 39: 983-990, 2008.

2. Omura A and Okita Y: Surgical treatment of thoracoabdominal aortic aneurysm. Kyobu Geka 65: 67-79, 2012.

3. Xing $\mathrm{B}$, Chen $\mathrm{H}$, Zhang $\mathrm{M}$, et al: Ischemic postconditioning inhibits apoptosis after focal cerebral ischemia/reperfusion injury in the rat. Stroke 39: 2362-2369, 2008.

4. Park ES, Gao X, Chung JM and Chung K: Levels of mitochondrial reactive oxygen species increase in rat neuropathic spinal dorsal horn neurons. Neurosci Lett 391: 108-111, 2006.

5. Varija D, Kumar KP, Reddy KP and Reddy VK: Prolonged constriction of sciatic nerve affecting oxidative stressors \& antioxidant enzymes in rat. Indian J Med Res 129: 587-592, 2009.

6. Ning N, Dang X, Bai C, Zhang C and Wang K: Panax notoginsenoside produces neuroprotective effects in rat model of acute spinal cord ischemia-reperfusion injury. J Ethnopharmacol 139: 504-512, 2012.

7. Oshio K, Binder DK, Yang B, Schecter S, Verkman AS and Manley GT: Expression of aquaporin water channels in mouse spinal cord. Neuroscience 127: 685-693, 2004.

8. Rash JE, Yasumura T, Hudson CS, Agre P and Nielsen S: Direct immunogold labeling of aquaporin-4 in square arrays of astrocyte and ependymocyte plasma membranes in rat brain and spinal cord. Proc Natl Acad Sci USA 95: 11981-11986, 1998.
9. Nesic O, Lee J, Ye Z, et al: Acute and chronic changes in aquaporin 4 expression after spinal cord injury. Neuroscience 143: 779-792, 2006

10. Saadoun S, Bell BA, Verkman AS and Papadopoulos MC: Greatly improved neurological outcome after spinal cord compression injury in AQP4-deficient mice. Brain 131: 1087-1098, 2008.

11. Hinson SR, McKeon A and Lennon VA: Neurological autoimmunity targeting aquaporin-4. Neuroscience 168: 1009-1018, 2010.

12. Nicaise C, Soyfoo MS, Authelet M, et al: Aquaporin-4 overexpression in rat ALS model. Anat Rec (Hoboken) 292: 207-213, 2009.

13. Radad K, Gille G, Liu L and Rausch WD: Use of ginseng in medicine with emphasis on neurodegenerative disorders. J Pharmacol Sci 100: 175-186, 2006.

14. Zhao J, Su C, Yang C, et al: Determination of ginsenosides Rb1, $\mathrm{Rb} 2$ and $\mathrm{Rb} 3$ in rat plasma by a rapid and sensitive liquid chromatography tandem mass spectrometry method: Application in a pharmacokinetic study. J Pharm Biomed Anal 64-65: 94-97, 2012.

15. Wu L, Jin Y, Yin C and Bai L: Co-transformation of Panax major ginsenosides $R b_{1}$ and $R g_{1}$ to minor ginsenosides $C-K$ and $F_{1}$ by Cladosporium cladosporioides. J Ind Microbiol Biotechnol 39: 521-527, 2012.

16. Lee JG, Baek SH, Lee YY, Park SY and Park JH: Anti-complementary ginsenosides isolated from processed ginseng. Biol Pharm Bull 34: 898-900, 2011.

17. Kwok HH, Guo GL, Lau JK, et al: Stereoisomers ginsenosides-20(S)- $\mathrm{Rg}_{3}$ and $-20(\mathrm{R})-\mathrm{Rg}_{3}$ differentially induce angiogenesis through peroxisome proliferator-activated receptor-gamma. Biochem Pharmacol 83: 893-902, 2012.

18. Yuan HD, Kim do Y, Quan HY, Kim SJ, Jung MS and Chung SH: Ginsenoside Rg2 induces orphan nuclear receptor SHP gene expression and inactivates GSK $3 \beta$ via AMP-activated protein kinase to inhibit hepatic glucose production in HepG2 cells. Chem Biol Interact 195: 35-42, 2012.

19. Ha SE, Shin DH, Kim HD, et al: Effects of ginsenoside Rg2 on the ultraviolet B-induced DNA damage responses in HaCaT cells. Naunyn Schmiedebergs Arch Pharmacol 382: 89-101, 2010.

20. Jiang JW, Chen XM, Chen XH and Zheng SS: Ginsenoside Rg3 inhibit hepatocellular carcinoma growth via intrinsic apoptotic pathway. World J Gastroenterol 17: 3605-3613, 2011.

21. Poon PY, Kwok HH, Yue PY, et al: Cytoprotective effect of 20S-Rg3 on benzo[a]pyrene-induced DNA damage. Drug Metab Dispos 40: 120-129, 2012

22. Lee KT, Jung TW, Lee HJ, Kim SG, Shin YS and Whang WK: The antidiabetic effect of ginsenoside Rb2 via activation of AMPK. Arch Pharm Res 34: 1201-1208, 2011.

23. Quan LH, Min JW, Sathiyamoorthy S, Yang DU, Kim YJ and Yang DC: Biotransformation of ginsenosides Re and Rg1 into ginsenosides $\mathrm{Rg} 2$ and $\mathrm{Rh} 1$ by recombinant $\beta$-glucosidase. Biotechnol Lett 34: 913-917, 2012.

24. Cheng B, Li J, Du J, Lv X, Weng L and Ling C: Ginsenoside Rb1 inhibits osteoclastogenesis by modulating NF- $\kappa \mathrm{B}$ and MAPKs pathways. Food Chem Toxicol 50: 1610-1615, 2012.

25. Tan S, Zhou F, Yu Z, et al: Study on characteristics of energy metabolism in skeletal muscle of rats with postoperative fatigue syndrome and interventional effect of ginsenoside Rb1. Zhongguo Zhong Yao Za Zhi 36: 3489-3493, 2011 (In Chinese).

26. Liu DH, Chen YM, Liu Y, et al: Rb1 protects endothelial cells from hydrogen peroxide-induced cell senescence by modulating redox status. Biol Pharm Bull 34: 1072-1077, 2011.

27. Lin ZY, Chen LM, Zhang J, et al: Ginsenoside Rb1 selectively inhibits the activity of L-type voltage-gated calcium channels in cultured rat hippocampal neurons. Acta Pharmacol Sin 33: 438-444, 2012.

28. Zhu J, Jiang Y, Wu L, Lu T, Xu G and Liu X: Suppression of local inflammation contributes to the neuroprotective effect of ginsenoside Rb1 in rats with cerebral ischemia. Neuroscience 202: 342-351, 2012.

29. Xia R, Zhao B, Wu Y, et al: Ginsenoside Rb1 preconditioning enhances eNOS expression and attenuates myocardial ischemia/reperfusion injury in diabetic rats. J Biomed Biotechnol 2011: 767930, 2011.

30. Bowes MP, Masliah E, Otero DA, Zivin JA and Saitoh T: Reduction of neurological damage by a peptide segment of the amyloid beta/A4 protein precursor in a rabbit spinal cord ischemia model. Exp Neurol 129: 112-119, 1994. 
31. Basso DM, Beattie MS, Bresnahan JC, et al: MASCIS evaluation of open field locomotor scores: effects of experience and teamwork on reliability. Multicenter Animal Spinal Cord Injury Study. J Neurotrauma 13: 343-359, 1996.

32. Livak KJ and Schmittgen TD: Analysis of relative gene expression data using real-time quantitative PCR and the 2(-Delta Delta C(T)) method. Methods 25: 402-408, 2001.

33. Nesic O, Guest JD, Zivadinovic D, et al: Aquaporins in spinal cord injury: the janus face of aquaporin 4. Neuroscience 168: $1019-1035,2010$
34. Bloch O, Papadopoulos MC, Manley GT and Verkman AS: Aquaporin-4 gene deletion in mice increases focal edema associated with staphylococcal brain abscess. J Neurochem 95: 254-262, 2005

35. Li L, Zhang H, Varrin-Doyer M, Zamvil SS and Verkman AS: Proinflammatory role of aquaporin-4 in autoimmune neuroinflammation. FASEB J 25: 1556-1566, 2011. 